\title{
Microstructure-Based Modeling of Metal Plasticity and Electron Microscopy Characterization of Automotive Structural Metals at Multi-Scales
}

\author{
Raja K Mishra
}

GM R\&D Center, Warren, MI USA.

Mechanical behavior of structural materials used in automobiles are influenced by their composition and microstructure as well as the strain path they are subjected to. Developing lighter vehicles to lower greenhouse gas emission requires timely development of new technology that also meets consumer demands for performance, safety and styling features at affordable prices. Deep knowledge of factors that influence the deformation mechanisms of metallic alloys at the atomic scale, lattice scale, grain scale and continuum scale are indispensable for fast and robust development of such new technology and electron microscopy based methods such as atomic resolution imaging, electron tomography, electron backscatter diffraction, conventional TEM, STEM and SEM imaging, etc. have acquired great significance in this new paradigm. Approaches that can take advantage of integrated computational materials engineering (ICME) tools require quantitative microstructural data in three and four dimensions (space and time) both as inputs and for validation [1]. Fig. 1 shows a schematic representation of the hierarchical approach that can be taken in a multi-scale approach in automotive manufacturing.

Fig. 2 shows the nucleation and growth of twinning studied by electron backscatter diffraction (EBSD) method in an extruded magnesium rod and how the texture of the material changes with the amount of deformation. Such information are important for validating crystal plasticity models that must correctly predict the changes in orientation and morphologies of grains if they are to be used in computer aided engineering of automotive components. Nanopillar indentation tests inside a TEM [2] can provide quantitative value of CRSS for alloys (after taking size effect into account) for such crystal plasticity model development. The lower yield strength and higher ductility of $\mathrm{Mg}-0.5 \% \mathrm{Ce}$ alloy extrusions in this study arises mainly due to a different texture of the starting material, which is related to the ease of twinning in $\mathrm{Mg}$ compared to $\mathrm{Mg}$-Ce during deformation, as confirmed by nanopillar compression tests $[2]$.

The ability to inform crystal plasticity models with quantitative microstructural information from electron microscopy based tools at different length scales makes it possible not only to do more accurate constitutive modeling of forming but also helps design materials with specific microstructural attributes [3].

\section{References:}

[1] PE Krajewski et al, JOM, 63 (2011), p. 24.

[2] J Ye, RK Mishra, AK Sachdev and AM Minor, Scripta Materialia, 64 (2011), p. 292.

[3] The authors acknowledge Li Jin for her help with in-situ EBSD study and Jia Ye for help with the nanopillar indentation of $\mathrm{Mg}$. 


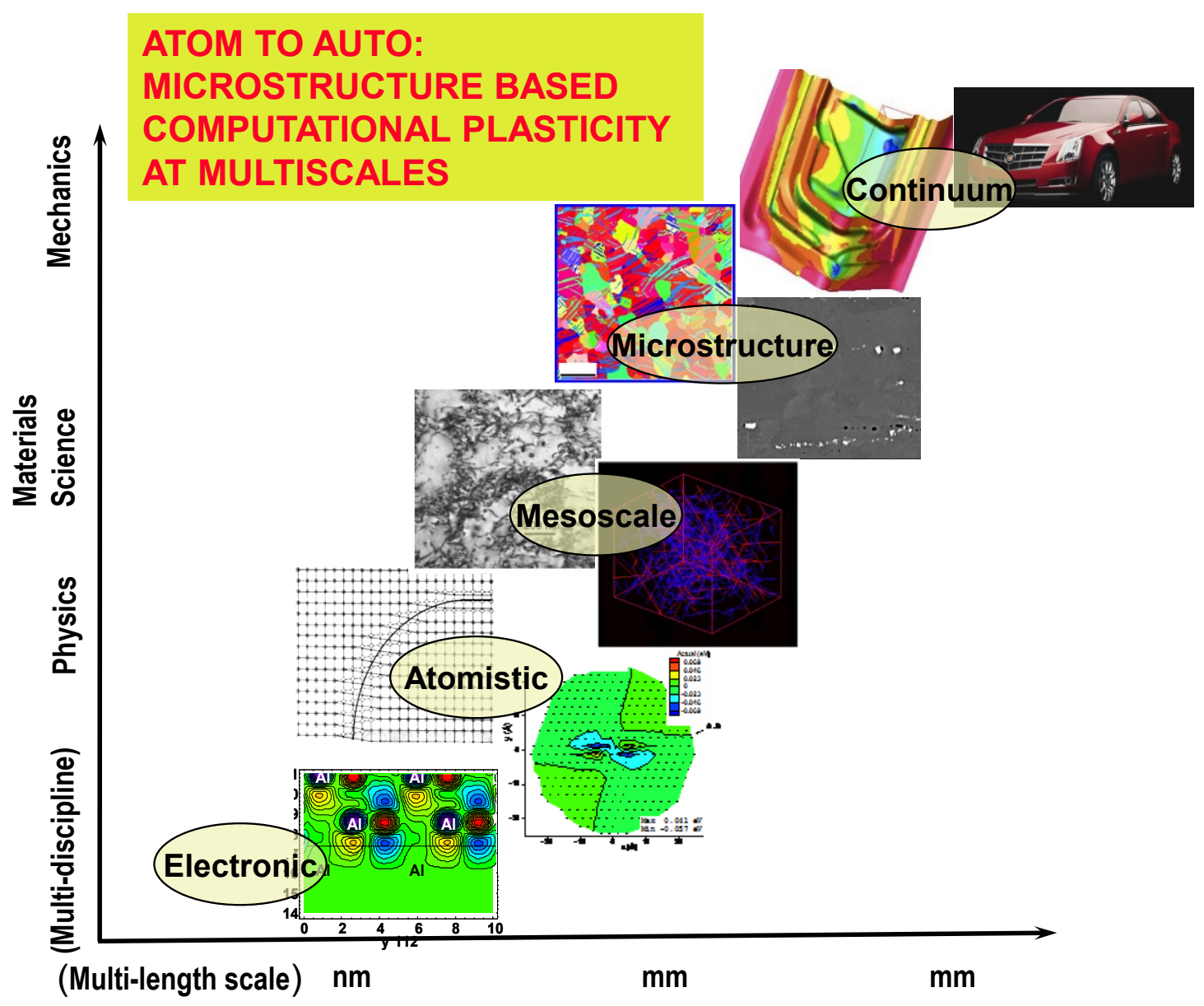

Figure 1. Schematic representation of the use of electron microscopy data at different length scales coupled with computational materials science for development of automotive materials.

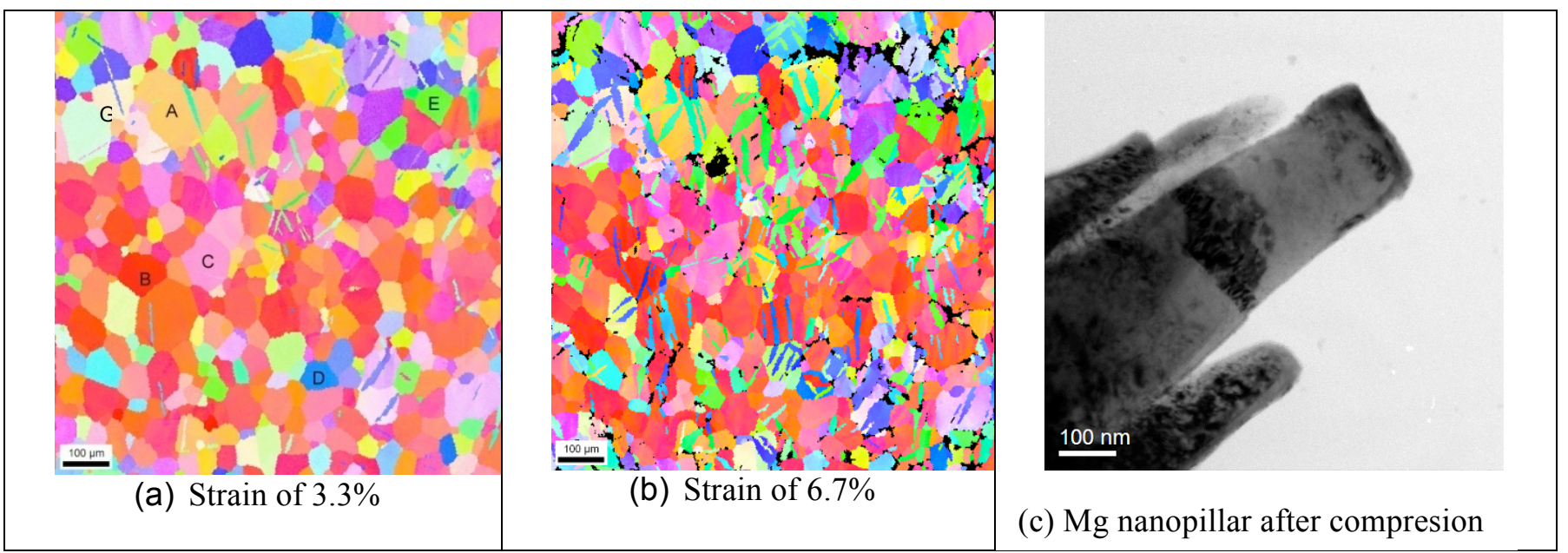

Figure 2. EBSD inverse pole figure map of formation of twins during in-situ compression of extruded magnesium rod inside the SEM. Note the changes in color of the twinned crystal in grain A due to $86^{\circ}$ rotation of the lattice by twinning and the increasing number of twins in the grains as deformation proceeds from $3.3 \%$ in (a) to $6.7 \%$ in (b). (c) Compressed nanopillar of Mg in TEM showing twinning. 